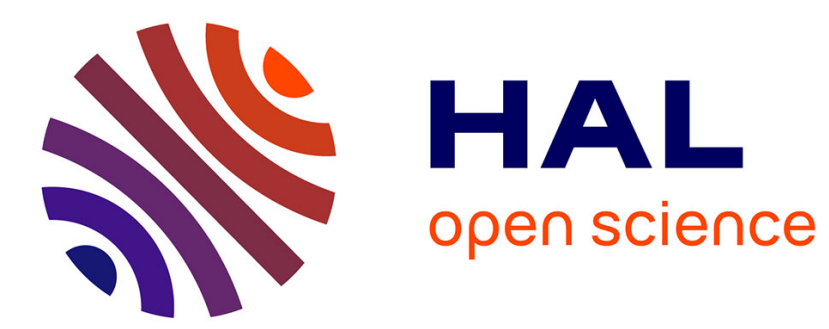

\title{
SPECTRAL PROPERTIES OF BILLIARDS AND QUANTUM CHAOS
}

\author{
C. Schmit
}

\section{To cite this version:}

C. Schmit. SPECTRAL PROPERTIES OF BILLIARDS AND QUANTUM CHAOS. Workshop on Semiclassical Methods in Nuclear Physics, 1984, Grenoble, France. pp.C6-379-C6-386, 10.1051/jphyscol:1984645 . jpa-00224247

\section{HAL Id: jpa-00224247 https://hal.science/jpa-00224247}

Submitted on 1 Jan 1984

HAL is a multi-disciplinary open access archive for the deposit and dissemination of scientific research documents, whether they are published or not. The documents may come from teaching and research institutions in France or abroad, or from public or private research centers.
L'archive ouverte pluridisciplinaire HAL, est destinée au dépôt et à la diffusion de documents scientifiques de niveau recherche, publiés ou non, émanant des établissements d'enseignement et de recherche français ou étrangers, des laboratoires publics ou privés. 


\title{
SPECTRAL PROPERTIES OF BILLIARDS AND QUANTUM CHAOS
}

\author{
C. Schmit \\ Division de Physique Thérique*, Institut de Physique Nucléaire, \\ B.P. 1, 91406 Orsay Cedex, Erance
}

\begin{abstract}
Résumé - Nous avons calculé les 800 premiers niveaux du stade pour chacun des 4 types de symétrie de la fonction d'onde. L'analyse des spectres obtenus montre que ceux-ci présentent la répulsion de niveaux et la très grande rigidité prévues par l'Ensemble Gaussien orthogonal de matrices aléatoires. Cette étude renforce la thèse selon laquelle ces propriêtés serajent caractëristiques des systèmes chaotiques quantiques.
\end{abstract}

Abstract - The first 800 eigenvalues of the stadium billiard have been evaluated numerically. It is shown that the four spectra obtained (corresponding to the four types of symmetry of the wave function) exhibit the fluctuation properties of the Gaussian Orthogonal Ensemble of Random Matrices. This reinforces the belief that these fluctuation properties are characteristic of quantum chaotic systems.

\section{I - INTRODUCTION}

In classical mechanics, the concept of chaotic systems is perfectly defined and the characteristic properties of such systems are known. In quantum mechanics, on the other hand, such a classification does not exist. One may obviously define a quantum system as being chaotic when its classical equivalent is chaotic. This is actually the definition of a quantum chaotic system. However, such a definition gives no information on what could be the characteristic properties of such a system. In this talk, we shall present some "experimental" results which may give an indication of what could be these characteristic properties. Following a somewhat historical order, we shall thus briefly recall some results of the random matrix theory and compare them to the available experimental data on nuclear resonances. Then we shall show (numerically) that the same fluctuation properties are found in some chaotic systems and not in integrable systems.

\section{II - PROPERTIES OF G.0.E. SPECTRA}

At low excitation energy, the nuclear spectra are rather well reproduced by shell model calculations. At present time such calculations are able to reproduce, at most, the first hundred levels of a nucleus, and it seems hopeless that a reliable description of higher excited states could be obtained. Furthermore such an exact description would not be very useful and one is more interested in statistical properties of the spectrum, such as the level density, which, in collision theory, are the only relevant quantities. Thus one is led to search for a statistical description of the nucleus. Such an approach, namely the random matrix theory, has been initiated by Wigner, and developed by Dyson and many other authors /1/. In this talk we shall concentrate on the Gaussian Orthogonal Ensemble (G.O.E.) which apply to systems which are invariant under rotation and time reversal. With these two conditions, one may show that the matrix ensemble consists of real symmetric matrices. If one add the condition that the matrix elements are statisticaliy independent, the probability density of an Hamiltonian of the ensemble is found to be :

\footnotetext{
*Laboratoire Associē au C.N.R.S.
} 


$$
p(H) \propto \exp \left\{-\operatorname{TrH}^{2} / 4 a^{2}\right\}
$$

which leads to the following probability density of eigenvalue :

$$
p\left(\varepsilon_{1}, \ldots, \varepsilon_{N}\right) \propto \exp \left(-\frac{1}{4 a^{2}} \sum_{1}^{N} \varepsilon_{K}^{2}\right) \prod_{i<j}\left|\varepsilon_{i}-\varepsilon_{j}\right|
$$

where $N$ is the dimension of the matrix. Given this joint probability, one may obtain the eigenvalue density by integrating eq. (II) over all but one variables.

$$
p(\varepsilon)=\int d \varepsilon_{2} \ldots d \varepsilon_{N} p\left(\varepsilon, \varepsilon_{2}, \varepsilon_{3}, \ldots, \varepsilon_{N}\right) \text {. }
$$

Similarly, correlation between eigenvalues may be obtained by evaluating the 2-points, 3-points, ... functions which are obtained from eq. (II) by integrating over all but two, three, ... variables. These integrations are far from obvious, and the mathematical technics needed to obtain them have been developed by Mehta, and here we shall only present the results.

\section{a) One-point function :}

The level density obtained from eq. (II) is given asymptotically by the semi circle law of Wigner :

$$
p_{N}(\varepsilon) \underset{N \rightarrow \infty}{\longrightarrow} \frac{1}{\pi a \sqrt{N}} \sqrt{1-\frac{\varepsilon^{2}}{4 a^{2} N}}
$$

This level density is obviousty far from experimental densities, but this point is not very important since we are mainly interested in fluctuations of the actual level density around the average one. In order to make the study of fluctuations and correlations easier, we shall work on what is called an unfolded spectrum, i.e. on a spectrum of constant average spacing. When the averaged level density is known, the unfolded spectrum $E_{K}$ may easily be obtained from the exact one $\varepsilon_{K}$ by the following relation :

$$
E_{K}=N \int_{-\infty}^{\varepsilon_{K}} p_{N}(\varepsilon) d \varepsilon \text {. }
$$

It is easy to show that the spectrum so obtained have an average spacing between adjacent levels equal to 1 , and in all the following we shall assume that we deal with such a spectrum.

$$
\text { b) Two-points function : }
$$

The exact expression of the joint probability density $p\left(E, E^{\prime}\right)$ has been given by Mehta and Gaudin. However this expression is not very practical to use, and we shal1 only give results on various quantities which are derived from this two-points function and which are easily measurable on a given spectrum. The first such quantity is the probability density of spacing between adjacent levels. In the GOE, it is found that this density $p(s)$ is very close to the Wigner surmise : 


$$
p(s)=\frac{\pi}{2} s e^{-\frac{\pi}{4} s^{2}} .
$$

The exact expression of the spacing distribution is almost indistinguishable from eq. $(V)$, the main difference being a slight modification of the variance $\left(\sigma_{G O E}^{2}=\right.$ $\left..286, \sigma_{1}^{2}=.277\right)$. The form of the spacing distribution given in eq. (V) has two importaft consequences on the spectrum. First, one may see that there is quite a strong level repulsion in the GOE spectrum. Indeed, on a sample of 1000 levels, one will find, on the average, only 8 spacings smaller than .1. This is to be compared to what would be observed in a Poisson spectrum where the spacing distribution would be :

$$
p(s)=e^{-s}
$$

In such a spectrum, the average number of spacings smaller than .1 would be close to 100 on a sample of 1000 levels. As for the large spacings, one also notes that in a sample of 1000 levels of a GOE spectrum, one witT find on $1 y$ I spacing larger than 3 , whereas in a Poisson spectrum the expected number of spacings larger than 3 would be 50 .

Thus the GOE spectrum seems, in some sense, far more rigid than a Poisson spectrum, since for the former small and large spacings are strongly forbidden as compared to the latter. As we shall see now, this rigidity of the spectrum is the most striking feature of GOE spectrum. Indeed, the evaluation of the 2-points function allows us not only to evaluate the spacing distribution eq. (V) but al so to evaluate correlations between spacings. In fact, in a Poisson spectrum (typically, radioactive decay) there are no correlation between spacings so that if one measure the length of an interval of $n$ spacings, $i$.e. the length $L_{n}=\left(E_{n+K}-E_{K}\right)$, the average length will be equal to $n$ and the variance of the length will also be equal to $n$. On the contrary, in a GOE. spectrum one finds strong correlations between spacings, specially for adjacent spacings for which the correlation coefficient is equal to - .273. More generally, one finds that the correlation coefficient between unit spacings separated by $K$ spacings decreases as $1 . / K^{2}$. The existence of strong negative correlations between spacings reinforces the rigidity of the spectrum. In fact if one measure the length of an interval of $n$ spacings, one will find that the averaged length is equal to $n$, as in the Poisson case. However, for the variance of this length, one will find that it increases as $\log (n)$ and not as $n$. For instance, for $n=10$, one would observe a variance equal to 10 in the Poisson case, and a variance equal to .742 in the GOE case. Thus the fluctuations of this length are much larger in a Poisson spectrum than in a GOE spectrum, which is very much alike a regular and uniform spectrum.

In order to evaluate quantitatively this rigidity, Dyson and Mehta introduced the $\Delta_{3}$ or $\Delta^{*}$ test which are related to the two points function only and which measure the deviation of a given spectrum from a picket fence or uniform spectrum. This test has the following expression :

$$
\Delta_{n}^{*}=\frac{1}{n} \operatorname{Min}_{a, b} \sum_{k=N}^{N+n-1}\left(E_{K}-a k-b\right)^{2}
$$

One may show that for a Poisson spectrum, the average value of $\Delta_{n}^{*}$ is equal to $n / 15$. Whereas for GOE one obtains 
(VIII)

$$
\Delta_{n}^{*} \propto .1 \log n
$$

Thus, one would find respectively. $\Delta_{15}^{*}=1$ and $\Delta_{150}^{*}=10$ in a Poisson spectrum, and $\Delta_{15}^{*}=.188$ and $\Delta_{150}^{*}=.412$ in a GOE spectrum. Finally the variance of $\Delta_{n}^{*}$ which is related to the 2,3 and 4 points function may also be evaluated and it has been shown that for Poisson this variance increases as $n$ whereas for GOE this variance is almost independent of $n$ and asymptotically equal to .012 .

\section{III - ANALYSIS OF EXPERIMENTAL DATA}

After this brief review of the properties of GOE spectra, let us see if the available experimental data on neutron resonances exhibit the fluctuation properties predicted by the random matrix theory. Such an analysis has been performed by Haq, Pandey and Bohigas who used a set of 1407 resonance energies corresponding to 30 sequences of 27 nuclei $/ 2 /$. The results of their analysis are presented on figures $1 \mathrm{a}$ and $1 \mathrm{~b}$. On these figures, one may note the impressive agreement between the
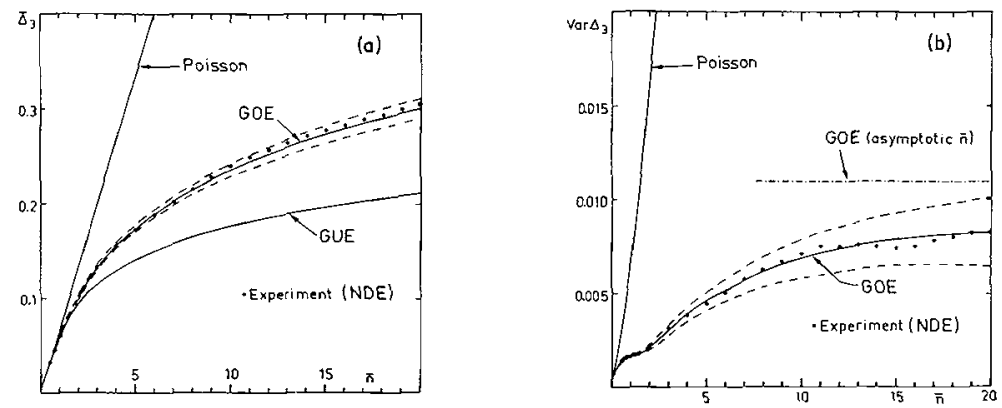

Fig. $1-\Delta_{3}(a)$ and $\sigma_{\Delta_{3}}^{2}$ (b) as a function of $n$. Experimental values are indicated by dots while the continuous 1 ines give the predictions for Poisson, G.0.E. and G.U.E.

theoretical values and the experimental ones for $\Delta_{3}(n)$ and for the variance of $\Delta_{3}(n)$. Thus, one may conclude that the GOE gives a good description of the fluctuation properties of nuclear spectra. However, this agreement raises immediately the question of why such a crude model, with so little physical input, (apart from rotational and time reversal invariances), is able to predict the experimental fluctuation properties. A possible answer to this question could be that these fluctuation properties reflect only the "complexity" of the physical system studied and that all systems with the same "complexity" would exhibit the same fluctuation pattern. However, this answer raises in turn another question, namely that of the definition of complexity of a quantum system. Is this "complexity" related to the large number of degrees of freedom as in the nuclear case, or is it related to the chaotic nature of the equivalent classical system ? 


\section{IV - CHAOTIC QUANTUM SYSTEMS}

In order to answer, at least partially, to the last question, we now turn to the study of quantum systems whose classical equivalent are chaotic. First let us recall that the classical conservative systems may be classified according to the properties of the time evolution of the system. Thus one may distinguish 5 different types of classical conservative systems (note, however, that almost no system enters in this classification) :

1) Integrable systems : a trajectory covers densely an $n$-dimensional torus in the 2n-dimensional phase-space (for instance, all systems with 1 degree of freedom, square or circular billiards in 2 dimensions).

2) Ergodic systems : aimost al1 trajectory densely covers the $(2 n-1)$ dimensional variety $s(E)$ in phase space, spending equal time in equal area.

3) Mixing systems : any area of $s(E)$ get spread uniformly over the energy surface $S(E)$ as $T \rightarrow \infty$. (Mixing $\Longrightarrow$ ergodic) (mixing $\Longleftrightarrow$ the system asymptotically forget its initial state).

4) K-systems : most initially close orbits separate exponentially with time $\mathrm{K}$-systems $\Longrightarrow$ mixing $\Longrightarrow$ ergodic. (Example : Arnold's cat, Sinai billiards, stadium, ....).

5) Bernouilli systems : sequential measurements of at least one observable are statisticaliy independent (ex.: Baker's transformation).

Thus we see from the examples given above that there exists classical systems which are simple enough so that one may be able to solve their quantum equivalent and which, nevertheless may be either integrable or chaotic. These simple systems are mainiy billiards with various frontiers. Their quantum equivalent amounts to find the vibration modes of a membrane fixed on a given contour $c$, i.e. to solve the Helmoltz equation :

$$
\Delta \Psi_{(r)}+k^{2} \Psi_{(r)}=0 \quad \vec{r} \in D
$$

$$
\Psi(\vec{r}) \longrightarrow 0 \quad \vec{r} \longrightarrow \mathrm{C}
$$

In order to solve this equation, we have settled a numerical program which is able to find rather efficiently the first 1000 levels of a given billiard. This program has been checked by applying it to integrable billiards (square and circular). This comparison has shown that the eigenvalues are determined with a precision better than $5 / 100$ of the average spacing. Besides, we found that in the circular case, the algorithm missed only 12 levels on the first 400 levels, and that this 12 levels correspond in fact to 12 pairs of almost degenerate levels that we could not separate. The analysis of the circular billiard data are presented on figures 2 and 3 where they are compared to the predictions of GOE and Poisson. This comparison, although not definitely concluding, shows nevertheless that for the circular bi1liard the spectrum is closer to a Poisson spectrum than to a GOE one. The spacing distribution shows indeed that smal1 and large spacings are rather frequent much more than in a GOE spectrum. As for the $\Delta^{*}$ values, al though smaller than the Poisson predictions, they are much larger than for GOE and the ir dependence on the number of levels is rather linear. The fact that the spectrum of an integrable system is of a Poisson type is not so surprising (al though not demonstrated). Indeed, for such a system, the spectrum is the sum of an infinite number of spectra, each corresponding to different values of the other quantum numbers. For instance, in the circular billiard, the spectrum is the sum of the spectra at fixed angular 
momentum. If one assumes that these different spectra are uncorrelated, then the sum of these spectra should give a Poisson spectrum.

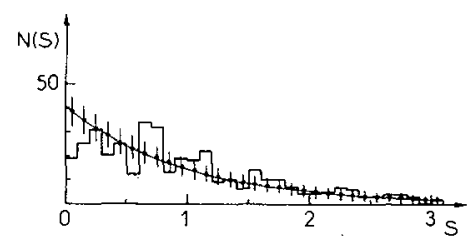

Fig. 2 - Level spacing distribution obtained with the first 400 levels of the circular billiard. Dots and error bars indicate the expected values and their standard deviations for Poisson.

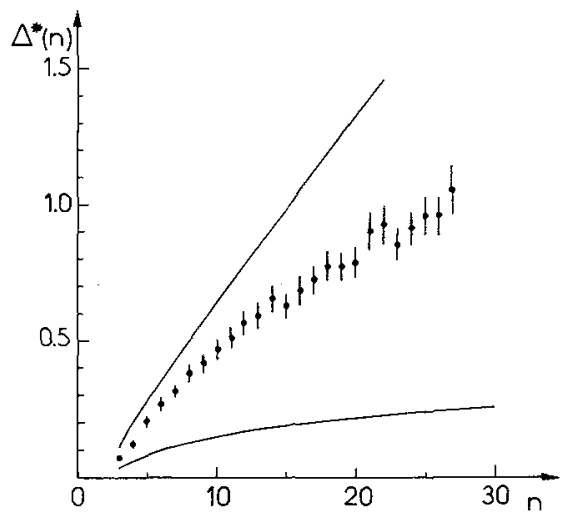

Fig. $3-\Delta^{*}$ as a function of $n$ for the circular billiard. "Experimental" values and their standard deviations are indicated by the dots and the error bars. The predictions for Poisson and G.O.E. are given by the continuous 1 ine.

Now that we have checked our algorithm, let us see the results obtained with a $\mathrm{K}$-system. We have applied our program to the stadium billiard and computed about 850 levels for each symmetry. The results of the analys is are presented on figures 4,5 and 6 . On figure 4 one may see that the spacing distributions are in very good agreement with the GOE predictions. As for the $\Delta^{*}$ values, they clearly exhibit a logarithmic behaviour with $n$, for values of $n$ as large as 100 or 150 , the agreement with GOE being rather good although slightly poorer. Similarly, the variance of $\Delta^{*}$ are found to be rather constant for $n$ smaller than 100 and not too far from the theoretical predictions. Thus although a more detailed analysis should be done, one may conclude that there are strong evidences that the spectrum of a $K$-system such as the stadium exhibits a strong rigidity and is indeed very close to a GOE spectrum. Note that we had aTready studied another K-system, namely the Sinai billiard and that this study had given the same conclusion although slightly less founded due to the poorer statistics $/ 3 /$. 


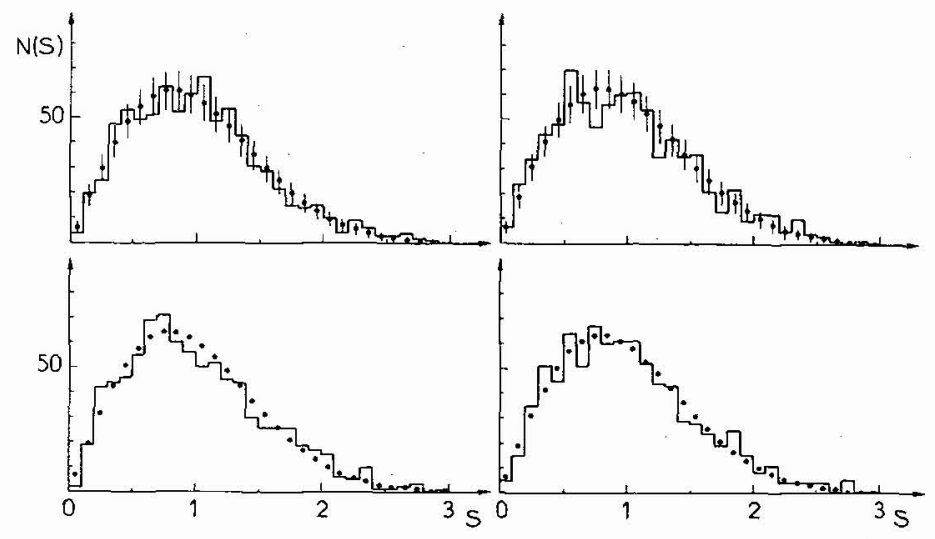

Fig. 4 - Spacing distributions obtained with the first 800 levels of the stadium, for each of the 4 symmetry types of the wave function. Dots and error bars (when indicated) give the G.0.E. predictions.

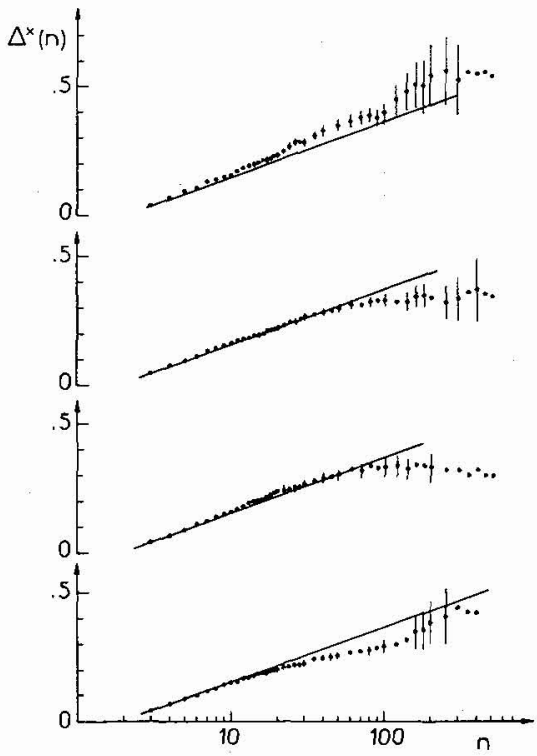

Fig. $5-\Delta^{*}$ as a function of $n$ for the stadium. Continuous $l$ ines give the G.O.E. predictions. Dots and error bars give the "experimental" values and their standard deviations. 


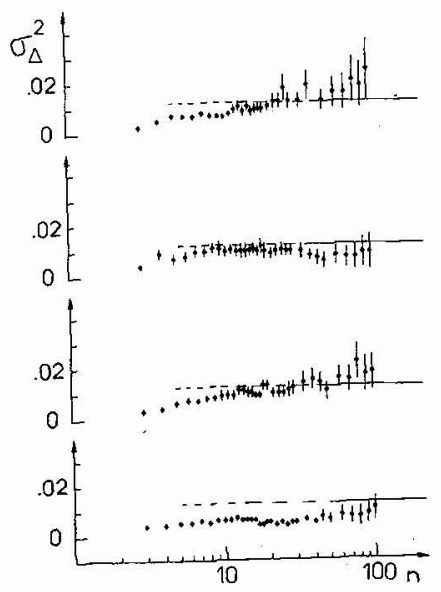

Fig. 6 - Same as fig. 5, for the variance of $\Delta^{*}$. The continuous 7 ine gives the G.0.E. asymptotic value.

\section{$V-$ CONCLUSION}

Thus, to summarize this talk, we may say that there are strong experimental evidences that nuclear spectra (at not too low an energy) are extremely rigid and that the same rigidity is observed in chaotic quantum billiards. On the contrary the spectrum of an integrable system appears to be much less rigid and close to a Poisson spectrum. A tentative interpretation of these "experimental" results could be that these fluctuation properties reflect the nature of the system studied and that the spectrum of a chaotic quantum system will exhibit the rigidity of GOE spectra. Such an interpretation is very appealing, however it is clear that more experimental data (nuclear and numerical) are needed to ascertain it and obviously a theoretical ground would be welcome.

The results presented here are part of a work done in collaboration with 0 . Bohigas and M.J. Giannoni.

\section{References :}

11/ Statistical Theories of Spectra : Fluctuations, edited by C.E. Porter (Academic, New York, 1965). M.L. MEHTA, Random Matrices and the Statistical Theory of Energy Levels (Academic, New York, 1967).

12) R.U. HAQ, A. PANDEY and O. BOHIGAS, Phys. Rev. Letters 48 (1982) 1086.

/3/ 0. BOHIGAS, M.J. GIANNONI, C. SCHMIT, Phys. Rev. Letters $\underline{52}$ (1984) 1. 